\title{
Improved visualization of breast cancer features in multifocal carcinoma using phase-contrast and dark-field mammography: an ex vivo study
}

\author{
Susanne Grandl ${ }^{1} \cdot$ Kai Scherer ${ }^{2}$ • Anikó Sztrókay-Gaul ${ }^{1}$ Lorenz Birnbacher ${ }^{2}$. \\ Konstantin Willer ${ }^{2} \cdot$ Michael Chabior $^{2}$. Julia Herzen ${ }^{2} \cdot$ Doris Mayr $^{3}$ • \\ Sigrid D. Auweter ${ }^{1} \cdot$ Franz $_{\text {Pfeiffer }}{ }^{2} \cdot$ Fabian Bamberg $^{4} \cdot$ Karin Hellerhoff $^{1}$
}

Received: 21 August 2014 / Revised: 19 December 2014 / Accepted: 7 April 2015 / Published online: 9 May 2015

(C) The Author(s) 2015. This article is published with open access at Springerlink.com

\begin{abstract}
Objectives Conventional X-ray attenuation-based contrast is inherently low for the soft-tissue components of the female breast. To overcome this limitation, we investigate the diagnostic merits arising from dark-field mammography by means of certain tumour structures enclosed within freshly dissected mastectomy samples.

Methods We performed grating-based absorption, absolute phase and dark-field mammography of three freshly dissected mastectomy samples containing bi- and multifocal carcinoma using a compact, laboratory Talbot-Lau interferometer. Preoperative in vivo imaging (digital mammography, ultrasound, MRI), postoperative histopathological analysis and ex vivo digital mammograms of all samples were acquired for the diagnostic verification of our results.

Results In the diagnosis of multifocal tumour growth, darkfield mammography seems superior to standard breast imaging modalities, providing a better resolution of small, calcified tumour nodules, demarcation of tumour boundaries with
\end{abstract}

Susanne Grandl and Kai Scherer contributed equally to this work.

Kai Scherer

kai.scherer@tum.de

1 Institute of Clinical Radiology, Ludwig-Maximilians-University Hospital Munich, Marchioninistrasse 15, 81377 Munich, Germany

2 Department of Physics and Institute of Medical Engineering, Technische Universität München, James-Franck-Strasse 1, 85748 Garching, Germany

3 Institute of Pathology, Ludwig-Maximilians-Universität München, Marchioninistr. 27, 81377 Munich, Germany

4 Department of Diagnostic and Interventional Radiology, University Hospital Tuebingen, Hoppe-Seyler-Strasse 3, 72076 Tuebingen, Germany desmoplastic stromal response and spiculated soft-tissue strands extending from an invasive ductal breast cancer.

Conclusions On the basis of selected cases, we demonstrate that dark-field mammography is capable of outperforming conventional mammographic imaging of tumour features in both calcified and non-calcified tumours. Presuming dose optimization, our results encourage further studies on larger patient cohorts to identify those patients that will benefit the most from this promising additional imaging modality.

Key Points

- X-ray dark-field mammography provides significantly improved visualization of tumour features

-X-ray dark-field mammography is capable of outperforming conventional mammographic imaging

- X-ray dark-field mammography provides imaging sensitivity towards highly dispersed calcium grains

Keywords Breast cancer $\cdot \mathrm{X}$-ray phase-contrast imaging . $\mathrm{X}$-ray phase-contrast imaging $\cdot \mathrm{X}$-ray dark-field imaging . Grating interferometry $\cdot$ Mammography

\section{Introduction}

With the implementation of nationwide screening programs in many countries, breast cancer mortality has steadily declined over the past decades [1]. Technical developments in conventional breast imaging have led to a significantly improved detection of early breast cancer and pre-invasive breast lesions. However, breast cancer remains the leading cause of cancer death in women worldwide [2] and underlying imaging techniques continue to have major limitations in daily clinical routine: while being the standard screening technique, the diagnostic sensitivity of conventional mammography 
remains fairly low, especially in women with dense breast tissue [3] and women at high familial risk of breast cancer $[4,5]$. Supplemental screening ultrasound is operatordependent and time-consuming [6]. MRI offers the highest diagnostic sensitivity for the detection of small cancers and has the advantage of avoiding ionizing radiation, but has low spatial resolution, is cost-intensive and requires the use of intravenous contrast agents [7].

Conventional X-ray mammography is based on attenuation-based contrast which is inherently low within breast soft tissue [8]. In the case of X-ray energies used in clinical mammography, phase-contrast and dark-field imaging are capable of overcoming these limitations as image formation in these modalities relies on the intrinsically enhanced electron density contrast and small-angle scattering capability of the tissue, respectively [8-10]. Besides, dark-field imaging benefits from sub-resolution sensitivity [11], which enables the detection of microstructures within the breast that are smaller than the pixel pitch of the detector $[12,13]$. With the recent introduction of a grating-based imaging approach utilizing a Talbot-Lau interferometer, retrieval of phasecontrast and dark-field signal is no longer restricted to highly brilliant X-ray sources, but is now compatible with clinical X-ray sources $[14,15]$. First studies investigating mastectomy samples with laboratory-based phase-contrast mammography showed promising results with respect to diagnostic potential by means of improved tumour visualization [16], image quality [17] and enhanced detection of ultra-small microcalcifications [12, 13].

In this work, we present the first specific case study of dark-field and absolute phase-contrast mammography conducted with two scanning directions using freshly dissected mastectomy specimens containing bi- and multifocal breast cancer (Table 1). We demonstrate that dark-field imaging alone results in improved visualization of tumour-infiltrated soft-tissue strands, multifocality, tumour boundaries and small calcified tumour clusters yet undifferentiated with conventional imaging techniques. Our results demonstrate the diagnostic merit of phase-contrast mammography for these specific tumour characteristics and encourage further efforts towards a rapid implementation into clinical routine.

\section{Materials and methods}

\section{Study design and sample acquisition}

The study was conducted in accordance with the Declaration of Helsinki and was approved by the local ethics committee (Ethikkommission of the Ludwig-Maximilian-University, Munich, project number 240-10, date of permission 26/08/ 2010 , amendment 30/05/2012). The three selected patients suffering from multifocal breast cancer were chosen from a collective of a prospective study on ex vivo phase-contrast mammography. From September 2012 to March 2013, all patients undergoing mastectomy were asked to take part in the study. All participants gave written informed consent before inclusion after adequate explanation of the study protocol. Indication for breast surgery followed recommendation of the interdisciplinary tumour conference. Inclusion criteria were a histologically proven breast cancer in preoperative core biopsy with a recommendation for mastectomy according to gynaecological guidelines or the patient's wish for mastectomy as well as completed preoperative conventional breast diagnostics (mammography, ultrasound with or without MRI).

\section{Preoperative diagnostics}

Preoperative diagnostics included clinical breast examination, clinical standard two-view digital mammography in craniocaudal (CC) and mediolateral-oblique (MLO) projections (Hologic Selenia Dimensions, Bedford, USA) using a standard breast compression paddle and high resolution
Table 1 Tumour characteristics of patients 1-3: histological diagnosis and tumour type; classification of mammographic breast density according to the American College of Radiology (ACR); preoperative imaging according to the Breast Imaging Reporting and Data System (BIRADS); dimensions and weight of the breast samples; maximum tumour size according to histopathology; TNM classification

\begin{tabular}{|c|c|c|c|c|c|c|}
\hline Patient & Histological diagnosis & ACR & BIRADS & $\begin{array}{l}\text { Sample dimensions } \\
\text { and weight }\end{array}$ & $\begin{array}{l}\text { Histological maximum } \\
\text { tumor diameter }\end{array}$ & TNM \\
\hline 1 & $\begin{array}{l}\text { Multifocal invasive carcinoma of no } \\
\text { special type (NST, formerly invasive } \\
\text { ductal), G1 }\end{array}$ & II & IV & $\begin{array}{l}16.5 \times 10.5 \times 9 \mathrm{~cm}^{3} \\
501 \mathrm{~g}\end{array}$ & $40 \mathrm{~mm}$ & $\mathrm{pT} 2(\mathrm{~m}) \mathrm{pN} 1 \mathrm{a}(1 / 16) \mathrm{Mx}$ \\
\hline 2 & $\begin{array}{l}\text { Trifocal invasive carcinoma of no special } \\
\text { type (NST, formerly invasive ductal) } \\
\text { and invasive lobular carcinoma, G1-2 }\end{array}$ & II & IV & $\begin{array}{l}25 \times 18 \times 4.5 \mathrm{~cm}^{3} \\
1009 \mathrm{~g}\end{array}$ & $15 \mathrm{~mm}$ & pT1c (trifocal) pN2a (7/16) Mx \\
\hline 3 & $\begin{array}{l}\text { Bifocal carcinoma of no special type } \\
\text { (NST, formerly invasive ductal) G2 }\end{array}$ & II & $\mathrm{V}$ & $\begin{array}{l}26 \times 13.5 \times 2.5 \mathrm{~cm}^{3} \\
570 \mathrm{~g}\end{array}$ & $25 \mathrm{~mm}$ & $\mathrm{pT} 2(\mathrm{~m}) \mathrm{pN} 0 \mathrm{Mx}$ \\
\hline
\end{tabular}

Note that the smallest sample diameter is measured in antero-posterior direction and does not correspond to the maximum craniocaudal diameter used for the experimental mammography 
B-mode ultrasound (standard linear transducer 13.5 MHz, Siemens Acuson Antares, Siemens Healthcare, Germany). In one patient additional MRI was performed by using a dedicated sensitivity-encoding-enabled bilateral breast coil with a 3.0-Tesla system (Magnetom Verio, Siemens Healthcare, Germany).

\section{Ex vivo clinical mammography}

The mastectomy samples were intraoperatively marked with surgical sutures for 3D orientation. The samples were fixed in craniocaudal position within a metal-framed sample holder to afford adequate breast compression. In order to ensure comparability, both a digital ex vivo mammography at a clinical mammography unit (Hologic Selenia Dimensions, Bedford, USA) with a pixel size of $70 \times 70 \mu \mathrm{m}^{2}$ and an experimental phase-contrast mammography with an effective pixel size of $85 \times 85 \mu^{2}$ were conducted without changing the sample position within the sample holder.

\section{Phase-contrast mammography setup}

A three-grating Talbot-Lau interferometer consisting of a conventional X-ray tube, a source grating $G_{0}$, a phase grating $G_{1}$ and an analyser grating $\mathrm{G}_{2}$ (Microworks $\mathrm{GmbH}$, Germany) was used for the experimental measurements. The source grating provides a spatially coherent X-ray beam, the phase grating generates an interference pattern downstream of the beam and the analyser grating detects changes of the latter, when a specimen is introduced into the beam, by which retrieval of phase- and dark-field contrast is practicable. A compact layout (distance $\mathrm{G}_{0}-\mathrm{G}_{1} 1 \mathrm{~m}$ and $\left.\mathrm{G}_{1}-\mathrm{G}_{2} \quad 0.5 \mathrm{~m}\right)$ in the third Talbot order was chosen using a Varian Paxscan 2520D flat panel with Gadox screen and $127 \times 127 \mu \mathrm{m}^{2}$ pixel size (effective pixel size of $85 \times 85 \mu \mathrm{m}^{2}$ ). The sample was positioned $2.7 \mathrm{~cm}$ upstream of $\mathrm{G} 1$. The Nonius FR 591 rotating anode X-ray tube (molybdenum target) was set to $40 \mathrm{kVp} / 70 \mathrm{~mA}$ (detailed description in [18]).

\section{Data acquisition}

A field of view of $12.8 \times 12.8 \mathrm{~cm}^{2}$ was achieved by stitching together $4 \times 4$ single projections.

Each scan was conducted with nine phase steps and an exposure time of $9 \mathrm{~s}$ each. Case study 1 and 3 were conducted with a bidirectional scanning approach, for the purpose of providing full detection sensitivity and absolute phase images as demonstrated in [18]. The phase image shown in case study 2 was obtained by a regularized 1D integration [19]. In order to increase image sharpness, phase images were high-frequency boosted.

\section{Dose}

The average glandular radiation dose of the in vivo mammography automatically registered by the mammography unit was 0.40 and $0.41 \mathrm{mGy}$ for sample $1,1.21$ and $1.27 \mathrm{mGy}$ for sample 2 and 1.35 and 1.26 mGy for sample 3 (mediolateral oblique projection and craniocaudal projection, respectively). The average glandular radiation dose of the ex vivo clinical mammography was $0.56 \mathrm{mGy}$ for sample $1,1.22 \mathrm{mGy}$ for sample 2 and $1.18 \mathrm{mGy}$ for sample 3 . The radiation dose of the experimental mammography was approximated by determining the incident air kerma $(2.1 \mathrm{mGy} / \mathrm{s})$ with a Dosimax plus/RQX detector system. The mean glandular dose was calculated by weighting this value with a Monte Carlo-based conversion factor of $0.389-0.422$, determined by the halfvalue layer $(\mathrm{Al})$ of $0.8 \mathrm{~mm}$ and breast thicknesses ranging from 4.5 to $5.0 \mathrm{~cm}[20,21]$.

\section{Histological workup}

Image data acquisition was followed by standard histological workup according to histopathological diagnostic guidelines. The mastectomy samples were fixated in a $4 \%$ neutralbuffered formaldehyde solution immediately after image acquisition. The formaldehyde-fixated samples were cut into 5-mm slices. Representative tissue sections were dehydrated in an ascending alcohol series before embedding in hot paraffin wax. The paraffin blocks were cut into 5- $\mu \mathrm{m}$ sections using a standard microtome and sections were stained with haematoxylin and eosin using standard protocols.

\section{Correlation phase-contrast mammography, ex vivo mammography, in vivo imaging and histology}

Phase-contrast and dark-field mammography of each sample were compared with preoperative in vivo standard imaging in consensus by three radiologists experienced in breast diagnostics who were blinded for the histopathological diagnosis. Afterwards, the findings were correlated with corresponding histological sections by a pathologist specialized in breast diagnostics.

\section{Results}

\section{Dark-field mammography reveals radiographically undetectable tumour nodules}

Patient 1 presented with a histologically proven recurrent breast carcinoma of the contralateral left breast after breastconserving therapy (data not shown). Additionally, two nodular indurations of the right breast in the upper lateral quadrant 
Fig. 1 Dark-field mammography reveals radiographically undetectable tumour nodules. Clinical ex vivo mammography in craniocaudal (cc) projection at $31 \mathrm{kVp}, 45 \mathrm{mAs}$ and $0.56 \mathrm{mGy}$ average glandular dose (silver filter) (a), experimental absorption-contrast mammography (abs-Mx, b), phase-contrast mammography (PC-Mx, c) and dark-field mammography (DF-Mx, d) of patient 1 at $40 \mathrm{kVp}, 70 \mathrm{~mA}$ and 66 mGy mean glandular dose (per scan direction); representative section from the in vivo MRI (e) including contrast-enhanced $\mathrm{T} 1$ weighted gradient-echo sequence after manual injection of $0.2 \mathrm{ml} / \mathrm{kg}$ meglumine gadopentetate (Magnograf ${ }^{\circledR} 0.5 \mathrm{mmol} / \mathrm{ml}$ ) (e, left) and the corresponding first subtraction image after $2 \mathrm{~min}$ $(\mathbf{e}, r i g h t)$ in an axial view using a dedicated sensitivity-encodingenabled bilateral breast coil with a 3.0-Tesla system (voxel size $0.7 \times 0.7 \times 2.0 \mathrm{~mm}$ ), field of view $360 \mathrm{~mm}$; base resolution 512; flip angle $15^{\circ}$; repetition time $6.5 \mathrm{~ms}$; echo time $2.47 \mathrm{~ms}$. Ultrasound (f); vertical line plots quantifying superior depiction of tumor (TU) 1,2 and 3 in DF-Mx (g, bottom) in comparison with abs-Mx (g, top) as indicated by arrows; in vivo mammography of patient 1 in cc projection at $29 \mathrm{kVp}, 11$ $\mathrm{mAs}$ and $0.41 \mathrm{mGy}$ average glandular dose (aluminium filter) (h); exemplary histological image (haematoxylin-eosin staining) of one calcified tumour nodule (i and $\mathbf{j}$ ), arrows indicating microcalcifications. The rectangles in $\mathbf{a}-\mathbf{d}$ and $\mathbf{h}$ indicate TU 1-3. The crossed arrows in b-d indicate bidirectional measurements
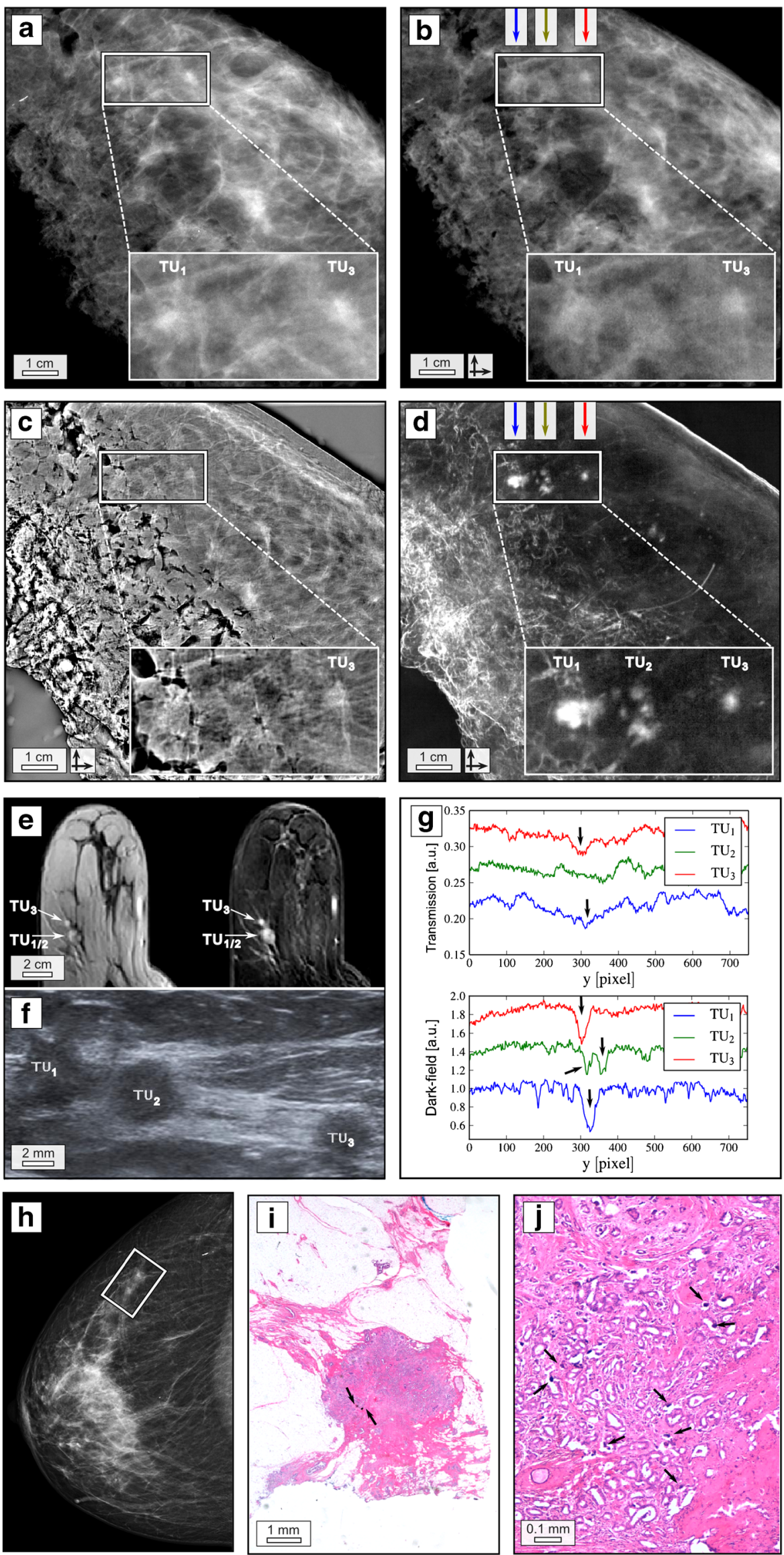
were clinically suspicious. Conventional in vivo mammography (IV-Mx) of the right breast revealed two corresponding adjacent lesions (Fig. 1h, white box) measuring 5 and $2 \mathrm{~mm}$ in the right upper lateral quadrant. Ultrasound revealed altogether seven suspicious lesions of 3 to $8 \mathrm{~mm}$ diameter in the upper lateral right quadrant three of which are shown in Fig. 1f. Dynamic MRI showed two suspicious enhancing lesions in the upper lateral quadrant of the right breast corresponding to the in vivo mammography (Fig. 1e). Histopathology of the whole breast revealed a multifocal carcinoma of no special type (NST, formerly invasive ductal carcinoma) grade 1 with the single tumor nodules measuring up to $6 \mathrm{~mm}$ and a maximum total diameter of $4 \mathrm{~cm}$ within an extensively growing ductal carcinoma in situ $(6 \mathrm{~cm})$.

Figure 1a-d shows ex vivo clinical mammography (EV$\mathrm{Mx}$ ), experimental absorption-contrast mammography (abs$\mathrm{Mx}$ ), phase-contrast mammography (PC-Mx) and dark-field mammography (DF-Mx) of the breast specimen 1 in craniocaudal orientation, which support the mammographic findings of two separated tumour nodules (TU1 and TU3), although being partially superimposed by surrounding breast parenchyma and resection margins. Surprisingly, in the DFMx (see Fig. 4a for the respective low-dose image) five additional smaller spots of high scattering contrast, composing a third nodule (TU2), were detected between the mammographically verified nodules TU1 and TU3. The spatial arrangement of the additional strongly scattering nodules within the DF image is consistent with the corresponding ultrasound section, showing a hypoechogenic "bridge" connecting the very lateral TU1 and TU2 as well as the third nodule TU3. The detection of an additional, intermediate nodule may explain why the first nodule TU1 is presented distinctively enlarged within the MRI in comparison to TU3: the low spatial resolution of MRI in combination with a continuous perfusion of contrast agent within TU1 and TU2 seems to depict the latter falsely as one persuasive tumour mass. Histopathological workup (Fig. 1i, j) proved the nodules with high DF contrast to be small calcified tumour nodules of $6 \mathrm{~mm}$ maximum diameter. Since the overall volume of microcalcified tissue is small, linear extinction coefficient (EV-Mx, abs-Mx) and electron density (PC-Mx) of the tumorous tissue are hardly altered by the presence of calcium, which leads to a visual blurring of the tumour within the surrounding breast parenchyma. However, imaging sensitivity in DF-Mx is enhanced towards ultra-small calcifications in the size range of some microns, accounting for the strong scattering signal provided by microcalcified structures within the tumour nodules. The surrounding breast parenchyma does not contribute to the scattering signal, which enables a clear differentiation of the small calcified tumour nodules from the surrounding breast tissue. Vertical line plots in abs-Mx (Fig. 1g, top) and DF-Mx (Fig. 1g, bottom) visualize the superior depiction of all tumour nodules by means of DF-Mx. Contrast-to-noise ratios (difference between signal intensities of tumour and respective surrounding tissue, divided by the standard deviation of the pure image noise) of 14.28 (TU1), 4.82 (TU2) and 7.76 (TU3) in the DF-Mx by far exceed those in the abs-Mx of 3.58 (TU1), 1.71 (TU2) and 4.14 (TU3). In the presented case, DF-Mx was the only radiographic imaging modality depicting and detecting additional tumour nodules correctly, yet undetectable or spatially unresolved within conventional mammography and MRI.

\section{Dark-field mammography reveals pervasion of the breast with partially tumorous soft-tissue strands}

Patient 2 presented with a palpable mass in the upper lateral quadrant of the left breast. IV-Mx (Fig. 2e) shows a mass lesion of $7 \mathrm{~mm}$ diameter in the upper lateral quadrant of the left breast associated with a suspicious architectural distortion. Ultrasound showed a corresponding suspicious architectural distortion measuring $14 \times 12 \times 8 \mathrm{~mm}^{3}$ with typical posterior acoustic shadowing. Histopathology of the whole breast revealed a trifocal carcinoma with the single tumours lying directly adjacent to each other including an intermediate grade (G2) carcinoma NST and an intermediate to low grade (G2-3) invasive lobular carcinoma.

Figure 2a-d shows EV-Mx, abs-Mx, PC-Mx and DF-Mx of sample 2. Histological images in haematoxylin-eosin staining show parts of the tumour-infiltrated tissue strands originating from the main tumour (Fig. 2f, g). Spiculated softtissue strands originating from the tumour and perfusing the surrounding tissue within a distance of approximately $2 \mathrm{~cm}$ were detected in all image modalities. However, in the DF$\mathrm{Mx}$, the extension of the strands was distinctively vaster than suggested by the other image modalities, even reaching up to the posterior resection margin (inlays in Fig. 2d). Histology revealed that the tissue strands consist of vessels and are partially infiltrated by tumour cells. The poor differentiability of the spiculated strands in IV-Mx, EV-Mx, abs-Mx and PC-Mx within the surrounding tissue can mainly be attributed to the small diameter (1-1.5 mm) of the strands in comparison to the underlying breast tissue $(5 \mathrm{~cm})$ in the beam direction as well as the fact that the differences in electron density between the tissue strands and the surrounding fatty breast tissue are relatively low. Hence the effective change in linear extinction coefficient (EV-Mx, abs-Mx) and electron density (PC-Mx) induced by the tissue strands with respect to the embedding tissue is minor, resulting in a poor contrast which is insufficient for proper delineation of the tissue strands from the surrounding tissue $(\mathrm{CNR}=0.86)$. However, the scattering properties within the strands strongly differ from the surrounding tissue. By means of DF-Mx, a clear differentiation $(\mathrm{CNR}=3.98)$ from the surrounding tissue was achieved, because scatter caused by the vessels and cellular structures within strands is not limited by their thickness. Although the 

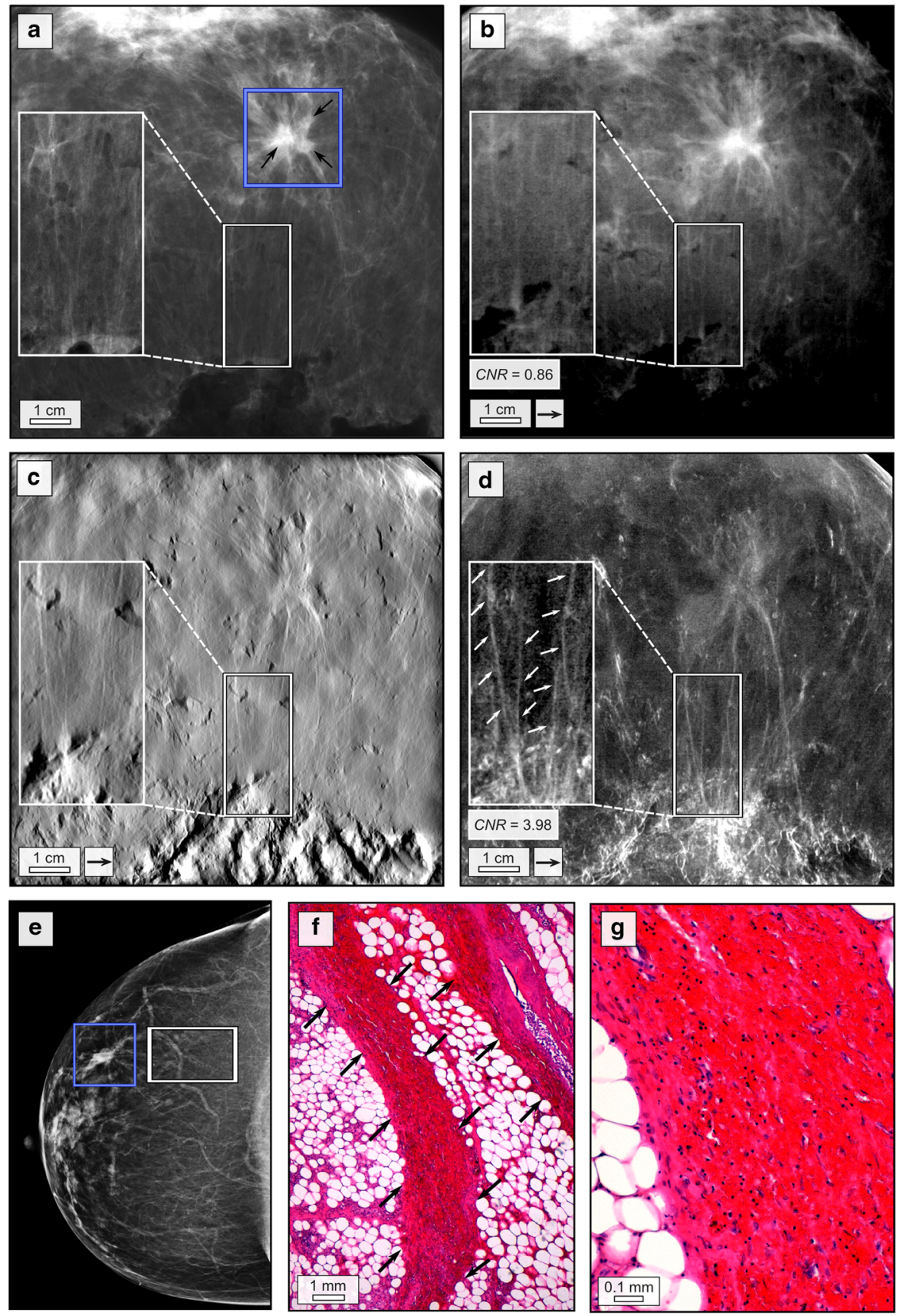

Fig. 2 Dark-field mammography reveals pervasion of breast with partially tumorous soft-tissue strands. Clinical ex vivo mammography in craniocaudal (cc) projection at $29 \mathrm{kVp}, 120 \mathrm{mAs}$ and $1.22 \mathrm{mGy}$ average glandular dose (rhodium filter) (a), experimental absorptioncontrast mammography (b), phase-contrast mammography (c) and dark-field mammography (d) at $40 \mathrm{kVp}, 70 \mathrm{~mA}$ and $66 \mathrm{mGy}$ mean glandular dose (per scan direction); in vivo mammography in cc projection at $29 \mathrm{kVp}, 105 \mathrm{mAs}$ and1.27 mGy average glandular dose (rhodium filter) (e) of patient 2 in craniocaudal projection; blue rectangles in a and $\mathbf{e}$ indicate the trifocal carcinoma; white rectangles in a-e indicate partially infiltrated tissue strands emerging from the tumour; exemplary histological image (haematoxylin-eosin staining) of partially tumour-infiltrated tissue strands (f) and (g) indicated by arrows. The arrows in $\mathbf{b}-\mathbf{d}$ indicate one-directional measurements micromorphology of the strands remains spatially unresolved, sub-resolution sensitivity towards the inherent structures is provided by the dark-field signal. Thus, the small-angle scattering signal caused by vascular walls and structures with high cellularity is detected by DF-Mx. Since inherent structures are mostly aligned alongside the strands, scatter is directed unidirectionally resulting in the adding up of signal. Besides, fatty tissue is hardly scattering, resulting in a flat background signal facilitating the depiction of the fine tumour strands from the surrounding tissue, yet undetectable in conventional imaging. 
With the information obtained by DF-Mx available in the preoperative mammogram of this patient, an additional preoperative MRI would likely have been recommended for clarifying the tumour extent with respect to pectoral muscle infiltration.

\section{Phase-contrast and dark-field mammography reveal tumour margins}

Patient 3 presented with a palpable mass in the upper lateral quadrant of the left breast. Palpation revealed two suspicious
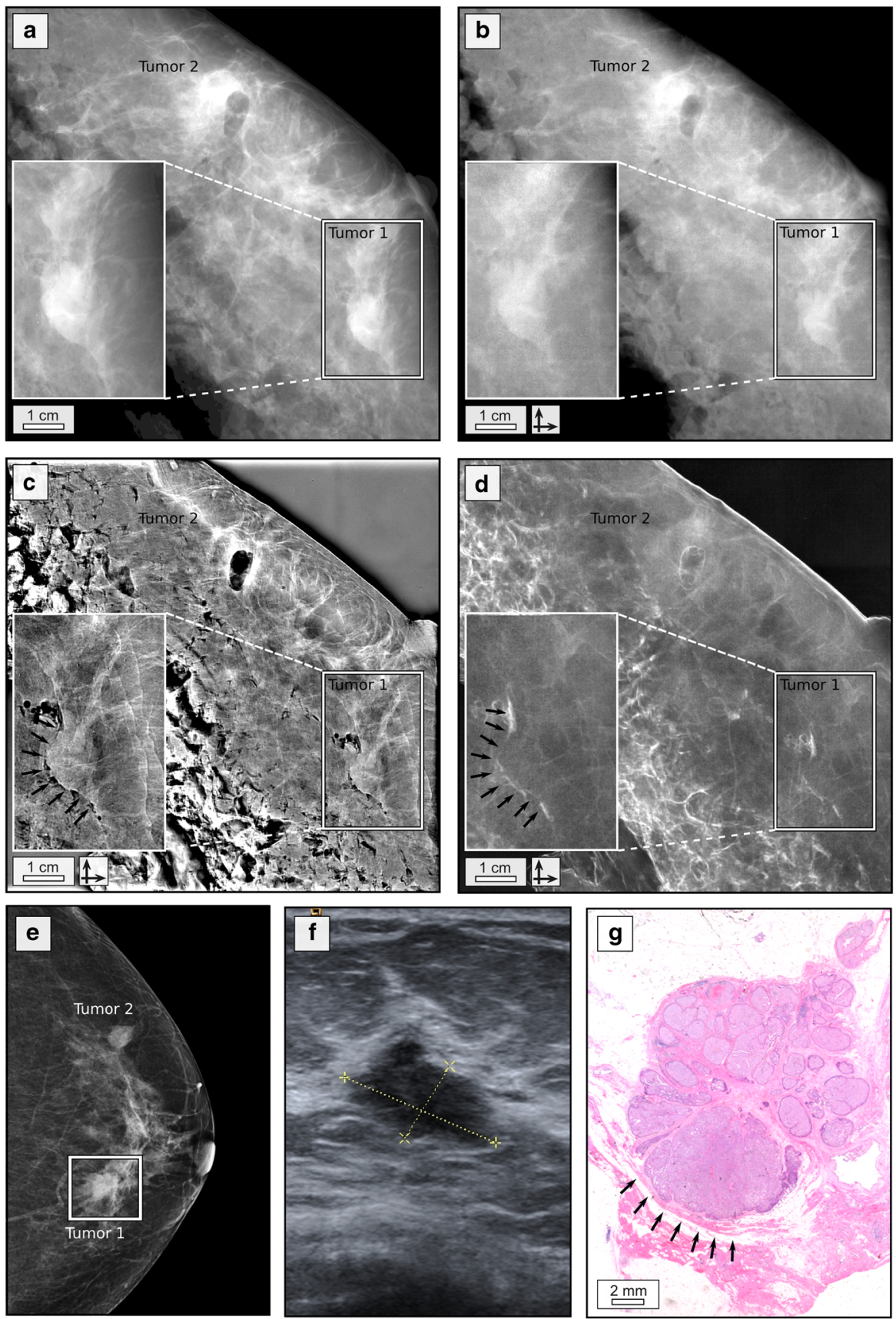

Fig. 3 Phase-contrast and dark-field mammography reveal tumour margins. Clinical ex vivo mammography in craniocaudal (cc) projection at $27 \mathrm{kVp}, 120 \mathrm{mAs}$ and $1.18 \mathrm{mGy}$ average glandular dose (rhodium filter) (a), experimental absorption-contrast mammography (b), phasecontrast mammography (c) and dark-field mammography (d) at $40 \mathrm{kVp}, 70 \mathrm{~mA}$ and $70 \mathrm{mGy}$ mean glandular dose (per scan direction); in vivo mammography in cc projection at $29 \mathrm{kVp}, 125 \mathrm{mAs}$ and

$1.26 \mathrm{mGy}$ average glandular dose (rhodium filter) (e) of patient 3 in craniocaudal projection; white rectangle indicating tumour 1; ultrasound of tumour 1 (f); histological image (haematoxylin-eosin staining) of tumour $1(\mathbf{g})$; the posterior margin of tumour 1 is indicated by black arrows in $\mathbf{c}, \mathbf{d}$ and $\mathbf{g}$. The crossed arrows in $\mathbf{b}-\mathbf{d}$ indicate bidirectional measurements 
indurations: in the left upper lateral and in the left lower medial quadrant. IV-Mx (Fig. 3e) revealed a suspicious mass (tumour 1), measuring $14 \times 9 \mathrm{~mm}^{2}$ in the left lower medial quadrant which was mainly superimposed by breast parenchyma. A second mass of $12 \times 8 \mathrm{~mm}^{2}$ was found in the left upper lateral quadrant (tumour 2). Sonographically (Fig. 3f shows tumour 1), both tumours had typical malignant features such as poor echogenicity, blurred margins and a hyperechogenic rim. Histopathology revealed tumour 1 (Fig. $3 \mathrm{~g}$ ) as a $2.5 \times$ $1.2 \times 1 \mathrm{~cm}^{3}$ moderately differentiated (G2) invasive carcinoma NST, tumour 2 as a partially haemorrhagic, $2.5 \times 2.3 \times 2 \mathrm{~cm}^{3}$ moderately differentiated (G2) invasive carcinoma NST containing a postbioptic haemorrhagic cavity.

Figure 3a-d shows EV-Mx, abs-Mx, PC- and DF-Mx of sample 3. Note the air inclusions representing the tissue defect at the biopsy site in tumour 2. Both tumour manifestations are detectable within the resected breast in all modalities. In the PC- and DF-Mx, an additional strong, linear contrast was observed exclusively at the dorsal margin of tumour 1 (black arrows in Fig. 3c, d), enabling a clear differentiation from the adjacent breast tissue. Histopathological workup revealed a desmoplastic stromal reaction and fibrous changes alongside the edge of the tumour resulting in a sharp demarcation from the surrounding tissue (black arrows in Fig. 3g). The distinct separation of tissue is accompanied by a strong local variation in refractive index across the tumour and adherent parenchyma, which generates a high contrast within the phase signal. Further X-rays are scattered at the tissue border alongside the tumour margin as visible within the dark-field image signal. Contrasting, absorption-based imaging (IV-Mx, EV-Mx and abs-Mx) is hardly sensitive towards this specific tumour entity and displays posterior and anterior tumour margins in a similar manner.

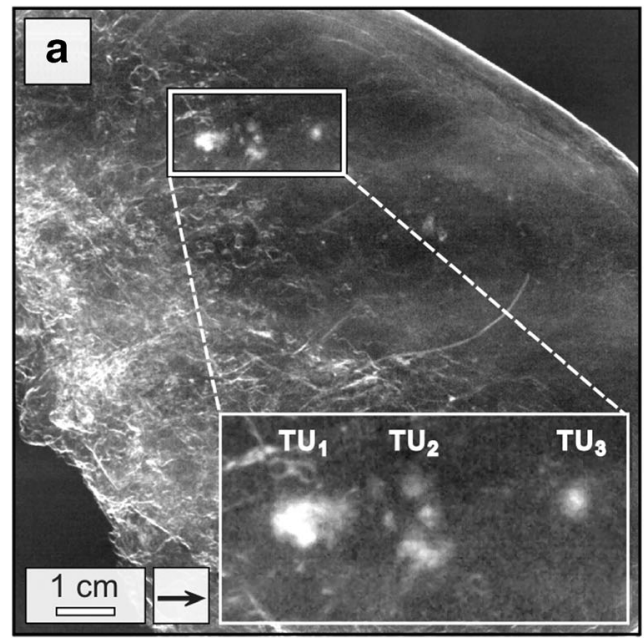

Fig. 4 Comparison of low- and high-dose dark-field mammography. Low-dose (22 mGy mean glandular dose) dark-field mammography of patient 1 conducted in one scan direction and $3 \mathrm{~s}$ exposure time per phase step (a) and corresponding high-dose measurements (66 mGy mean

\section{Discussion}

In this ex vivo study on three selected mastectomy samples containing bi- and multifocal carcinoma, we evaluated the diagnostic value of grating-based phase-contrast and darkfield mammography at a conventional, polychromatic X-ray source. We showed that dark-field mammography provides complementary information in comparison with conventional imaging, by revealing tissue strands emerging from the tumour, detecting microcalcified tumour nodules and depicting focality and tumour margins in correlation with histology.

Until now, only a few investigations have focused on the diagnostic value of dark-field imaging of dissected breast samples [12, 13, 15-17, 22]. Stampanoni et al. analysed five mastectomy samples using phase-contrast mammography and found that the fused images created from the grating-based absorption-, phase- and dark-field signals enable a better visualization of small tumours, skin infiltration and differentiation between scars, healthy tissue and invasive cancer [16]. A blinded, follow-up reader study came to the conclusion that the fused images outperform conventional digital mammograms in terms of overall image quality and sharpness [17]. Our results confirm these findings, indicating an improved visibility of high frequency features (tissue strands, tumour margins and spiculations) within the phase-contrast and dark-field mammograms. Moreover, we could demonstrate that sub-resolution dark-field sensitivity helps to detect highly dispersed microcalcifications when examining complete breast samples that are not visible in other modalities because of size and volume, but that are nonetheless indicative of malignancy. This is in accordance with studies by Anton and Michel et al. who analysed slices of cancer-bearing breast samples $[12,13]$ : they showed that the presence of

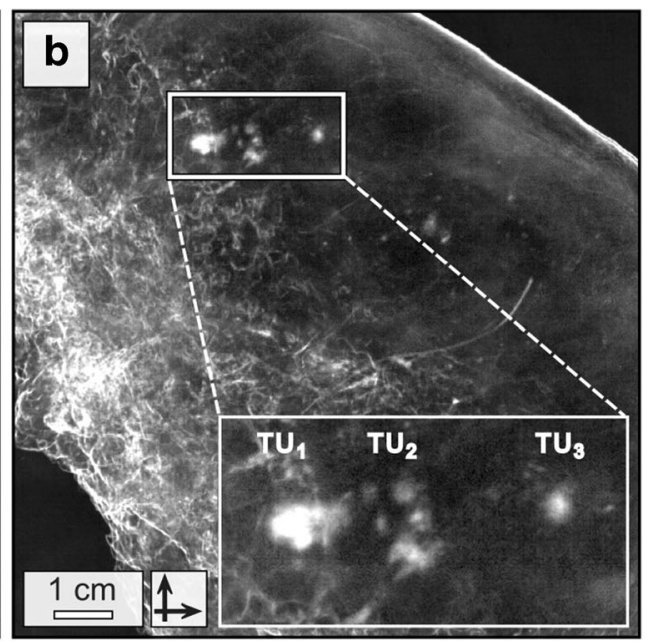

glandular dose per scan direction) conducted in two scan directions and $9 \mathrm{~s}$ exposure time per phase step (b) at $40 \mathrm{kVp} / 70 \mathrm{~mA}$, respectively. Both images offer equal quality in the detection of the tumour nodules 
microcalcifications within the tumour results in a high dark-field signal, although the microcalcifications were not detectable in the conventional mammogram.

Exceeding previously published studies on phase-contrast mammography on whole mastectomy samples, our study exemplarily indicates that dark-field mammography may outperform all conventional imaging modalities in the detection of small or low dense tumour nodules, fine structures and delineation of tumour tissue. The correct determination of tumour size and margins including tumour branches especially within dense breast tissue may prevent incomplete resection and the need for follow-up resections. Moreover, the capability of detecting minor or highly dispersed calcium grains strongly facilitates the sensitivity towards incipient calcificationprocesses and therewith detection of early stage tumours. To what extent the higher sensitivity in the detection of calcifications unresolved in conventional mammography will influence the percentage of overdiagnosis and lead to additional breast biopsies remains debatable.

One challenge in breast cancer treatment is the prevention of recurrent disease that can be caused by incomplete resection or unnoticed multifocality and multicentricity at the time of diagnosis. A considerable percentage (13-75\%) of primary breast cancers occur as multifocal disease with often small satellite nodules not detected in conventional breast imaging [23]. Breast MRI has been shown to be more sensitive in the detection of multifocality, but is time and cost intensive [23]. Our results indicate that dark-field mammography has the potential to detect small tumours, especially if microcalcified, at a better resolution than MRI, even though additional lesions - depending on their localization - might still need further histological assessment.

While the experimental measurements were conducted within a clinically feasible frame (compact setup, conventional $\mathrm{X}$-ray tube and adequate sample compression) the applied radiation dose exceeds the corresponding clinical ex vivo value (average glandular dose ranging from 0.56 to $1.22 \mathrm{mGy}$ ) by far. The estimated glandular dose applied at our experimental setup was 66-70 mGy (total exposure time of $81 \mathrm{~s}$ ) per projection and scan direction, depending on the sample thickness. Since measurements were not conducted dose-optimized, several aspects can be addressed to reduce the dose applied: an increase of currently $13.2-49.4 \%$ setup fringe visibility by improving the beam quality (design energy, beam filtration) and grating quality (duty cycle, grating substrate thickness, grating height) would imply an approximately 14-fold decrease in dose for phase-contrast imaging. An optimization of the detector with respect to the design energy of $27 \mathrm{keV}$ and the replacement of the X-ray target molybdenum with tungsten could further double the setup efficiency.

A decrease of 9 to $3 \mathrm{~s}$ exposure time per step would further decrease the dose by a factor of 3. Corresponding low-dose measurements were exemplarily conducted in case of patient
1 (one scan direction), providing equivalent quality of detection for the microcalcified tumour nodules (Fig. 4) and suggest that a much lower exposure time is practicable. These considerations would imply a reduction of dose down to $0.8 \mathrm{mGy}$ (1.6 mGy in the case of bidirectional imaging) which lies within the dose limits of $2.5 \mathrm{mGy}$ set by the European guidelines for mammography [24].

Currently phase-contrast imaging suffers from limited clinical experience and needs further technical improvements and dose optimization (the first dose-compatible measurements at $2.2 \mathrm{mGy}$ have been conducted with a revised setup recently). Previous studies demonstrate few selected cancer cases and, up to now, no independent multi-reader studies with larger patient cohorts and lower prevalence of malignancy have been performed. Thus, future studies will have to focus on the specific value of grating-based phase-contrast imaging for different tumour entities and on larger patient cohorts to identify those patients that will benefit the most from this promising additional imaging modality.

Acknowledgments The scientific guarantor of this publication is Karin Hellerhoff. The authors of this manuscript declare no relationships with any companies whose products or services may be related to the subject matter of the article. We acknowledge financial support through the DFG Cluster of Excellence Munich-Centre for Advanced Photonics (MAP), the DFG Gottfried Wilhelm Leibniz program and the European Research Council (ERC, FP7, StG 240142). Part of this work was carried out with the support of the Karlsruhe Nano Micro Facility (KNMF, www.kit.edu/knmf), a Helmholtz Research Infrastructure at Karlsruhe Institute of Technology (KIT). No complex statistical methods were necessary for this paper.

Institutional review board approval was obtained. The study was conducted in accordance with the Declaration of Helsinki and was approved by the local ethics committee. All participants gave written informed consent before inclusion after adequate explanation of the study protocol. Written informed consent was obtained from all subjects (patients) in this study. Parts of the image data shown in Fig. 2 were presented before by Scherer et al. (PLoS One 9(5): e93502, 2014) in a technical manuscript on two-directional X-ray phase-contrast mammography. However, the emphasis of the present study lies on different image features than presented in the previous study and the figure itself strongly differs from the one published before; so we are confident that all figures presented in the present manuscript meet the copyright criteria. Methodology: prospective, experimental, performed at one institution.

Open Access This article is distributed under the terms of the Creative Commons Attribution-NonCommercial 4.0 International License (http:// creativecommons.org/licenses/by-nc/4.0/), which permits any noncommercial use, distribution, and reproduction in any medium, provided you give appropriate credit to the original author(s) and the source, provide a link to the Creative Commons license, and indicate if changes were made.

\section{References}

1. Marmot MG, Altman DG, Cameron DA et al (2013) The benefits and harms of breast cancer screening: an independent review. Br J Cancer 108:2205-2240 
2. Ferlay J, Shin HR, Bray F et al (2010) Estimates of worldwide burden of cancer in 2008: GLOBOCAN 2008. Int J Cancer 127: 2893-2917

3. Pinsky RW, Helvie MA (2010) Mammographic breast density: effect on imaging and breast cancer risk. J Natl Compr Cancer 8: $1157-1164$

4. Schrading S, Kuhl CK (2008) Mammographic, US, and MR imaging phenotypes of familial breast cancer. Radiology 246:58-70

5. Tilanus-Linthorst M, Verhoog L, Obdeijn IM et al (2002) A BRCA1/2 mutation, high breast density and prominent pushing margins of a tumor independently contribute to a frequent falsenegative mammography. Int J Cancer 102:91-95

6. Moon HJ, Jung I, Park SJ et al (2014) Comparison of cancer yields and diagnostic performance of screening mammography vs. supplemental screening ultrasound in 4394 women with average risk for breast cancer. Ultraschall Med. doi:10.1055/s-0034-1366288

7. Sardanelli F, Giuseppetti GM, Panizza P et al (2004) Sensitivity of MRI versus mammography for detecting foci of multifocal, multicentric breast cancer in fatty and dense breasts using the whole-breast pathologic examination as a gold standard. AJR Am J Roentgenol 183:1149-1157

8. Keyrilainen J, Bravin A, Fernandez M et al (2010) Phase-contrast X-ray imaging of breast. Acta Radiol 51:866-884

9. Arfelli F, Bonvicini V, Bravin A et al (2000) Mammography with synchrotron radiation: phase-detection techniques. Radiology 215 : 286-293

10. Takeda T, Momose A, Ueno E et al (1998) Phase-contrast X-ray CT image of breast tumor. J Synchrotron Radiat 5:1133-1135

11. Yashiro W, Terui Y, Kawabata K et al (2010) On the origin of visibility contrast in $\mathrm{X}$-ray Talbot interferometry. Opt Express 18: 16890-16901

12. Anton G, Bayer F, Beckmann MW et al (2013) Grating-based darkfield imaging of human breast tissue. Z Med Phys 23:228-235
13. Michel T, Rieger J, Anton G et al (2013) On a dark-field signal generated by micrometer-sized calcifications in phase-contrast mammography. Phys Med Biol 58:2713-2732

14. Pfeiffer F, Weitkamp T, Bunk O et al (2006) Phase retrieval and differential phase-contrast imaging with low-brilliance X-ray sources. Nat Phys 2:258-261

15. Pfeiffer F, Bech M, Bunk O et al (2008) Hard-X-ray dark-field imaging using a grating interferometer. Nat Mater 7:134-137

16. Stampanoni M, Wang $Z$, Thuring $T$ et al (2011) The first analysis and clinical evaluation of native breast tissue using differential phase-contrast mammography. Investig Radiol 46:801-806

17. Hauser N, Wang Z, Kubik-Huch RA et al (2014) A study on mastectomy samples to evaluate breast imaging quality and potential clinical relevance of differential phase contrast mammography. Investig Radiol 49:131-137

18. Scherer K, Birnbacher L, Chabior M et al (2014) Bi-directional Xray phase-contrast mammography. PLoS One 9, e93502

19. Matias Di Martino J, Flores JL, Pfeiffer F et al (2013) Phase retrieval from one partial derivative. Opt Lett 38:4813-4816

20. Dance DR (1990) Monte Carlo calculation of conversion factors for the estimation of mean glandular breast dose. Phys Med Biol 35: 1211-1219

21. Dance DR, Skinner CL, Young KC et al (2000) Additional factors for the estimation of mean glandular breast dose using the UK mammography dosimetry protocol. Phys Med Biol 45:3225-3240

22. Wang Z, Hauser N, Singer G et al (2014) Non-invasive classification of microcalcifications with phase-contrast X-ray mammography. Nat Commun 5:3797

23. Bendifallah S, Werkoff G, Borie-Moutafoff C et al (2010) Multiple synchronous (multifocal and multicentric) breast cancer: clinical implications. Surg Oncol 19:e115-e123

24. Perry N, Broeders M, de Wolf C et al (2008) European guidelines for quality assurance in breast cancer screening and diagnosis. Fourth edition-summary document. Ann Oncol 19:614-622 